\title{
Association between Helicobacter pylori Infection and Idiopathic Thrombocytopenic Purpura in Adults
}

\author{
Takayoshi Suzuki , Masashi Matsushima, Atsushi Takagi and Tetsuya Mine
}

Department of Internal Medicine, Tokai University School of Medicine, Isehara, Kanagawa, Japan

"Corresponding author: Takayoshi Suzuki, Division of Gastroenterology \& Hepatology, Department of Internal Medicine, Tokai University School of Medicine, 259-1193, 143 Shimokasuya, Isehara, Kanagawa, Japan, Tel: 81-463-93-1121; E-mail: takayosh@is.icc.u-tokai.ac.jp

Rec date: 2014 Feb 10, Acc date: 2014 April 29, Pub date: 2014 May 07

Copyright: (C) 2014 Suzuki T, et al. This is an open-access article distributed under the terms of the Creative Commons Attribution License, which permits unrestricted use, distribution, and reproduction in any medium, provided the original author and source are credited.

\begin{abstract}
A Gram-negative spiral bacterium, Helicobacter pylori, has been known to be a causative agent of chronic gastritis and peptic ulcer disease and plays an important role in increasing the risk for the development of gastric cancer. In addition, the infection has also been implicated in the development of extragastric diseases, such as hematological, systemic, cardiovascular and neurological diseases. Accumulating evidence indicates that successful eradication of $H$. pylori results in an increase of the platelet count in approximately half of $H$. pylori-positive ITP patients. However, the efficacy of the eradication was quite different in ITP patients among different countries worldwide. In this review, we summarize the recent evidences with regard to the association between $H$. pylori infection and ITP. We also provide information about the possible pathogenetic mechanisms and the factors for predicting platelet recovery.
\end{abstract}

Keywords: Helicobacter pylori; Idiopathic thrombocytopenic purpura; Eradication therapy

\section{Introduction}

Helicobacter pylori (H. pylori) are a spiral-shaped microaerophilic Gram-negative bacterium that colonizes the human gastric mucosa, and is known as a causative agent of acute and chronic gastritis. Prolonged $H$. pylori infection leads to gastrointestinal diseases mediated by chronic inflammation, such as atrophic gastritis, peptic ulcer, gastric hyperplastic polyp, gastric cancer and mucosa-associated lymphoid tissue (MALT) lymphoma [1]. Although the immunological response to $H$. pylori infection has been suggested as a major factor involved in the gastric mucosal inflammation, it is still not clearly understood why $H$. pylori-infected individuals are at a high risk of development of such diverse diseases. Eradication of $H$. pylori has been shown to be effective for reducing the gastric mucosal damage and also reducing the incidence of peptic ulcer recurrence. Furthermore, it has been reported that more than half of the patients with MALT lymphoma are cured by successful eradication of $H$. pylori.

In addition, $H$. pylori has been implicated in the development of various systemic disorders, including autoimmune diseases such as idiopathic thrombocytopenic purpura (ITP), rheumatoid arthritis, Sjogren's syndrome, and Schonlein-Henoch purpura, as well as irondeficiency anemia, chronic urticaria, cardiovascular diseases, and neurological disorders [2]. However, because the number of reported cases is still few for many of these conditions, the relevance of $H$. pylori infection in the development of these disorders is yet to be firmly established.

Among the aforementioned diseases, however, accumulating evidence indicates that successful eradication of $H$. pylori results in an increase of the platelet count in approximately half of $H$. pyloripositive ITP patients. $H$. pylori eradication therapy has been recommended as a useful measure in treatment of ITP by both the Maastricht IV Consensus [3] and The Second Asia-Pacific Consensus Guidelines [4]. In this review, we will summarize the currently available evidence concerning the relationship between ITP and $H$. pylori infection.

\section{Basic Treatment of ITP}

$\mathrm{TP}$ is an acquired autoimmune disease in which the emergence of autologous antibodies directed against platelets triggers enhanced platelet destruction, resulting in thrombocytopenia. To establish a definite diagnosis of this disease, it is necessary to rule out other diseases that cause thrombocytopenia (e.g., collagen vascular diseases, malignant tumors, drug-induced or radiation-induced disorders, infectious diseases, coagulation abnormalities). ITP can be divided into acute and chronic types according to the clinical course of the illness. Acute ITP, which is more common in children, is characterized by a rapid onset and is associated with antecedent infection in about $80 \%$ of the pediatric patients. Spontaneous remission occurs at a frequency of more than $80 \%$ within 4 months after the onset. This disease entity has a good prognosis, with a final cure rate of $90 \%$. On the other hand, chronic ITP, which is more common in adults, is associated with prolonged thrombocytopenia that typically persists for more than 6 months after the onset. In these patients, spontaneous remission is extremely rare, and it is not unusual for the patients to suffer from repeated relapses even after the initiation of therapy $[5,6]$.

Because it is relatively rare for the sustained thrombocytopenia in these patients to result in fatal conditions such as intracranial hemorrhage, the conventional treatment for chronic ITP basically consists of observation of the course of illness. In the event of detection of a bleeding tendency or a decrease in the platelet count to $30,000 / \mu \mathrm{L}$ or lower, administration of corticosteroids or immunosuppressive drugs or splenectomy may be selected as the standard treatment. In the case of complications associated with childbirth or the need for surgical treatment of the patients for any 
Page 2 of 5

indication, platelet transfusion aimed at temporarily increasing the platelet count, with or without high-dose intravenous immunoglobulin therapy, is reported as a feasible treatment strategy [6].

More recently, new treatments including eradication of $H$. pylori, thrombopoietin receptor agonist therapy, and anti-CD20 antibody therapy have been introduced, and their efficacies have been documented in some studies [7-9].

\section{Therapeutic Efficacy of $\boldsymbol{H}$. pylori Eradication in ITP}

In 1998, Gasbarrini et al. [10] administered H. pylori eradication therapy to $11 \mathrm{H}$. pylori-positive patients with chronic ITP, and reported recovery of the platelet counts in all 8 patients in whom the bacterium had been successfully eradicated. On the other hand, no increase of the platelet count was observed in the remaining 3 ITP patients in whom the eradication therapy was unsuccessful or who $H$. pylori-negative. Thereafter, in 2001, Emilia et al. [11], who followed the study of Gasbarrini and colleagues, administered $H$. pylori eradication therapy to 13 patients with ITP who were $H$. pyloripositive, and observed recovery of the platelet count in 6 of these patients. Both of these studies were performed in Italy. Like Italy, Japan is among the countries with a high prevalence of $H$. pylori carriers. Various studies in Japan have revealed that the efficacy rate of $H$. pylori eradication in inducing recovery of the platelet count in $H$. pylori-positive ITP patients is approximately $50 \%$, which is comparable to the reports from Italy [12-17].

The following observations have been cited as evidence corroborating the causal relationship between $H$. pylori and chronic ITP:

1) Platelet count recovery did not occur or was not sustained in patients with ITP in whom $H$. pylori eradication was unsuccessful;

2) in patients in whom primary $H$. pylori eradication failed to increase the platelet count, successful secondary eradication resulted in recovery of the platelet counts;
3) Among patients who underwent no $H$. pylori eradication in prospective randomized trials, none showed any recovery of the platelet counts;

4) No recovery of the platelet counts occurred- in H.pylori-negative ITP patients who underwent eradication therapy [12-14].

While a number of reports have been published on the efficacy of $H$. pylori eradication in inducing the recovery of platelet counts in chronic ITP patients, Jarque et al. reported in 2001 that the platelet counts increased in only 3 of $23 \mathrm{H}$. pylori-positive ITP patients who received $H$. pylori eradication therapy, and that the efficacy rate of $H$. pylori eradication therapy in inducing platelet count recovery in Spain was only 5\% [18]. Michel et al. reported a similar frequency of $H$. pylori infection between ITP patients (29\%) and controls (29\%) in France, and that there were no significant differences in the clinical features between $H$. pylori-positive and $H$. pylori-negative patients with ITP (e.g., sex, age, frequency of hemorrhagic symptoms, platelet count at the time of diagnosis, anti-platelet antibody positivity rate, steroid response). Thus, they expressed doubts as to the direct involvement of $H$. pylori in the development of ITP [19]. In addition, the efficacy rates of $H$. pylori eradication in inducing platelet count recovery in ITP patients in the US, Serbia, and Mexico are also extremely low, similar to the case in Spain. On the other hand, a recent study conducted in Colombia revealed an extremely high prevalence of $H$. pylori infection (91\%), and a high percentage of patients $(83 \%)$ showing a favorable platelet response to $H$. pylori eradication therapy [20]. It remains unclear why there are variations in the platelet response to $H$. pylori eradication therapy in ITP patients among different countries worldwide (Table 1). One possibility is host factors, such as racial differences in the immune responses to the infection. Other explanations for the geographic discrepancy include bacterial factors and environmental factors. However, the precise factors have yet to be clarified.

\begin{tabular}{|l|l|l|l|l|}
\hline Study year, Reference & Country & $\begin{array}{l}\text { Mean Follow-up time } \\
\text { (Month) }\end{array}$ & Plate response (\%) & $\begin{array}{l}\text { Prevalence (\%) of H.pylori } \\
\text { infection }\end{array}$ \\
\hline Gasbarrini et al. [10] & Italy & 4 & $8 / 8(100)$ & $11 / 18(61)$ \\
\hline Emilia et al. [11] & Italy & 8.3 & $6 / 12(50)$ & $13 / 30(43)$ \\
\hline Jarque et al. [18] & Spain & 24 & $3 / 23(13)$ & $40 / 56(71)$ \\
\hline Kohda et al. [17] & Japan & 148 & $12 / 19(63)$ & $22 / 35(62)$ \\
\hline veneri et al. [32] & Italy & 11.7 & $11 / 15(73)$ & $25 / 35(71)$ \\
\hline Michel et al. [19] & USA & 11.5 & $4 / 14(29)$ & $16 / 74(22)$ \\
\hline Suzuki et al. [12] & Japan & 6 & $6 / 13(46)$ & $25 / 36(69)$ \\
\hline Fujimura et al. [14] & Japan & $12<$ & $88 / 161(55)$ & $300 / 435(69)$ \\
\hline Suvaidzic et al. [37] & Serbia & 18 & $6 / 23(26)$ & $39 / 54(72)$ \\
\hline Sayan et al. [38] & Turkey & 13 & $8 / 20(40)$ & $34 / 20(59)$ \\
\hline Campuzano-Maya et al. [20] & Colombia & 12.2 & $24 / 29(83)$ & $29 / 32(91)$ \\
\hline Estrada-Gomez et al. [39] & Mexico & 4.6 & $3 / 14(21)$ & $14 / 23(60)$ \\
\hline
\end{tabular}




\begin{tabular}{|l|l|l|l|l|}
\hline Sivapathasingam et al. [40] & Australia & 12.2 & $5 / 9(56)$ & $9 / 16(56)$ \\
\hline Joakson et al. [41] & Canada & 48 & $3 / 4(75)$ & $4 / 22(18)$ \\
\hline Tag et al. [42] & Korea & 57.6 & $25 / 17(68)$ & $23 / 25(92)$ \\
\hline Payandeh et al. [43] & Iran & 6 & $26 / 15(58)$ & $35 / 52(67)$ \\
\hline Gan et al. [44] & Malaysia & 6 & $3 / 10(30)$ & $11 / 50(22)$ \\
\hline
\end{tabular}

Table 1: Platelet response to $H$. pylori eradication therapy in ITP patients

While several studies have been conducted in various countries, as discussed above, we demonstrated the usefulness of $H$. pylori eradication in patients with ITP for the first time in a randomized controlled study carried out in 2005 [12]. More specifically, in our series, there were no cases in the non- $H$. pylori eradication group $(n=12)$ that showed recovery of the platelet counts at the measurement carried out 6 months after enrollment, whereas 46.2\% (including 6 cases showing a complete response and 4 showing a partial response) of patients in the $H$. pylori eradication group $(n=13)$ showed obvious platelet count recovery $(\mathrm{P}=0.02)$. Moreover, Asahi et al. administered $\mathrm{H}$. pylori eradication therapy to 37 patients with ITP (including $26 \mathrm{H}$. pylori-positive and $11 \mathrm{H}$. pylori-negative ITP patients), regardless of whether or not $H$. pylori infection had been detected. $H$. pylori eradication was successful in all the $26 \mathrm{H}$. pylori-positive patients, and increased of the platelet counts was observed in 16 (62\%) of these 26 patients; in contrast, none of the $H$. pylori-negative patients showed any platelet count increases [13]. Furthermore, Fujimura et al. reported the results of a large-scale multicenter retrospective study carried out in Japan [14]. According to their report, eradication therapy administered to 228 of $300 \mathrm{H}$. pylori-positive ITP patients, and eradication was successful in $78 \%$ of these cases. Platelet responses were observed in 88 (57\%) of the 155 patients who were followed up for at least 12 months. Furthermore, in 2007, Franchini et al. carried out a meta-analysis of 17 studies involving a total of 788 patients with ITP, and established the usefulness of $H$. pylori eradication therapy in inducing platelet count recovery in these patients [21]. In 2009, Stasi et al. analyzed $1555 \mathrm{H}$. pylori-positive patients with chronic ITP included in 25 studies, and reported that the percentage of patients showing platelet count recovery in response to eradication therapy was $50.3 \%$, with a tendency for the response rate to be higher in countries where the prevalence of $H$. pylori infection was higher [22]. As for the longterm prognosis of ITP patients after $H$. pylori eradication, the platelet responses were sustained at adequate levels for many years [23-25]. Adverse reactions to eradication therapy included mild abdominal pain, diarrhea and rash, which occurred in no more than $5 \%$ of the patients. None of these adverse reactions were serious, confirming the safety of this therapy.

\section{Distinctive Clinical Features and Predictors of the Platelet Responses to $\boldsymbol{H}$. pylori Eradication}

In ITP patients in whom eradication therapy was effective in inducing platelet count recovery, the PAIgG level and anti-platelet antibody titer were decreased after the treatment. Therefore, it was inferred that $H$. pylori infection may be involved in the production of the anti-platelet antibody, although the underlying mechanisms still remain rather unclear. Several hypotheses have been proposed to explain the mechanisms by which $H$. pylori may cause chronic ITP. On the basis of recent evidences, some plausible pathogenetic mechanisms have been proposed, including cross-reactivity between platelet glycoproteins and $H$. pylori CagA protein, genetic host factors and bacterial factors. In addition, clinical evidence is available regarding the predictors of a platelet count response to $H$. pylori eradication.

\section{Patient background factors}

The Patients' age, sex, history of transfusion, history of peptic ulcer, platelet count prior to eradication, and history of treatment prior to the eradication had no effect on the recovery of the platelet count in response to $H$. pylori eradication [12,14]. Accordingly, it is presumed that eradication therapy may be beneficial in patients with intractable ITP who show poor response to either steroid therapy or splenectomy. Sato et al. studied the effect of eradication therapy in 31 patients, and reported that the pepsinogen I/II ratio was significantly lower, and the endoscopic atrophy of the gastric mucosa was more severe, in patients who responded to the eradication therapy than in those who did not [26]. In addition, Fujimura et al. recommended early eradication therapy because they found significantly greater increases of the platelet counts after eradication in patients with shorter disease duration than in those with longer disease duration [14].

\section{Bacterial factors}

Immunological response to $H$. pylori can cause gastric mucosal inflammation, and initially consists of recruitment of polymorphonuclear leukocytes, followed by that of $\mathrm{T}$ and $\mathrm{B}$ lymphocytes, plasma cells, and macrophages, along with gastric epithelial cell damage. According to previous reports, the virulence factors of $H$. pylori are closely related to the development of diseases in the host via modulating the host immune response: $H$. pylori strains possessing both cagA and vacA genes have been linked to higher cytotoxicities; the CagA protein was translocated into the gastric epithelial cells by a type IV secretion system, eventually promoting epithelial cell proliferation [27]; VacA causes vacuolation of the target cells; vacA $\mathrm{s} 1 / \mathrm{ml}$ strains produce higher levels of the vacuolating cytotoxin than other strains, inducing a greater degree of inflammatory cell infiltration; iceA1 has also been recognized as another pathogenic factor and been suggested as a marker for peptic ulcer. Therefore, detailed examination of the virulence factors of $H$. pylori may be of importance for understanding the pathogenesis of chronic ITP $[28,29]$.

Takahashi et al. reported that anti-platelet antibodies show high cross-reactivity with the CagA protein [15]; Franceschi et al. also reported that anti-CagA antibody cross-reacted with platelet protein in patients with chronic ITP [30]. In our previous study, the serum antiCagA antibody titers were significantly higher in the platelet responders than those in the non-responders [12]. These studies 
Page 4 of 5

suggest the existence of a positive association between the platelet response to $H$. pylori eradication therapy and the presence of CagA. Our studies revealed that cagA, vacAs1, vacAm1, and iceA1were found similarly in both patients showing and not showing a platelet response to $H$. pylori eradication therapy, suggesting that these $H$. pylori virulence factors may not be related to the platelet recovery [12]. However, Emilia et al. compared the infecting strains obtained from ITP patients and those from control participants, and reported that the frequency of the $H$. pylori cagA gene and concomitant $H$. pylori cagA, vacAs1, and iceA genes was statistically significantly higher in the ITP patients than in the controls [23]. Further studies are needed to establish the clinical relevance of the $H$. pylori virulence factors in chronic ITP patients.

\section{Host factors}

Chronic persistent infection with $H$. pylori triggers increased production of various cytokines in the gastric mucosa [31]. Hashino et al. measured the serum levels of IL-2, INF $\gamma$, IL-6, IL-15, TPO, and TGF $\beta 1$ in patients with ITP to examine the differences between the patients showing and not showing a platelet response to $H$. pylori eradication therapy. They found that the blood TGF $\beta 1$ levels were significantly higher in platelet responders after successful $H$. pylori eradication [16]. Veneri et al. reported that $H$. pylori-postitive ITP patients showed a lower frequency of HLA-DRB1 ${ }^{\star} 03$ and higher frequency of HLA-DRB $1^{\star} 11$, HLA-DRB $1^{\star} 14$, and HLA-DQB ${ }^{\star} 03$ than $H$. pylori-negative patients [32]. Moreover, a HLA-DQB1 ${ }^{\star} 03$ pattern was associated with a higher probability of a platelet response to $H$. pylori eradication therapy.

Ishiyama et al., who carried out single-strand conformation polymorphism analysis of complementarity-determining region 3 (CDR3) of the T cell receptor $\beta$ chain gene of peripheral blood T cells, noted that $\mathrm{V} \beta 5.2, \mathrm{~V} \beta 15$, and $\mathrm{V} \beta 19$ gene usage by clonally expanded $\mathrm{T}$ cells in the peripheral blood obtained before the H. pylori eradication therapy was significantly higher in the platelet responders than in the non-responders or healthy controls. An abrogation of clonally expanded $\mathrm{T}$ cells was observed after eradication therapy in some responders. Based on these findings, they concluded that specific $\mathrm{T}$ cell clones were highly likely to play an important role in thrombocytopenia in $H$. pylori-related ITP patients [33]. We reported the importance of gene polymorphism of TNFB $(+252)$ as a predictor of the platelet recovery in ITP patients after $H$. pylori eradication. The prevalence of platelet responders in ITP patients with the G/G or G/A genotype of TNFB $(+252)$ was significantly higher than that in the ITP patients with the A/A genotype [34]. Asahi et al. have suggested that H. pylori infection may facilitate platelet destruction via the reduced levels of the inhibitory $\mathrm{F} c \gamma$ receptor IIB in peripheral blood monocytes, leading to the onset of ITP [35]. In addition, Yeh et al. reported that $H$. pylori IgG is required for bacteria to induce P-selectin expression and that a significant release of P-selectin is essential for $\mathrm{H}$. pylori to induce platelet aggregation, resulting in a decreased platelet count [36].

Since relatively small numbers of patients were recruited in the aforementioned trials, further studies involving larger numbers of patients will be needed to establish the findings as clinical evidence.

\section{Position and Future Perspectives of $H$.pylori Eradication Therapy in the Treatment of ITP}

Before publication of the studies indicating an association between H. pylori infection and thrombocytopenia, chronic ITP was considered to be an autoimmune disease of unknown etiology. It was commonly treated with steroids, splenectomy or high dose intravenous immunoglobulin, which do not give rise to cure of the ITP, but maintain the platelet count within a safe range. However, previous studies have shown that $H$. pylori eradication therapy is effective in inducing platelet recovery in about half of all $H$. pyloripositive chronic ITP patients, and its long-term efficacy and safety have also been confirmed. Thus, $H$. pylori eradication has become a standard first-line therapy for $H$. pylori-positive chronic ITP patients.

However, much remains unclear as to the etiologic mechanisms of $H$. pylori-related ITP, including the geographical discrepancies of the platelet responses after $H$. pylori eradication and inconsistencies among the predictors of the platelet responses to $H$. pylori eradication. Thus, further in vitro and clinical studies are required to address these issues.

\section{References}

1. Blaser MJ, Atherton JC (2004) Helicobacter pylori persistence: biology and disease. J Clin Invest 113: 321-333.

2. Tan HJ, Goh KL (2012) Extragastrointestinal manifestations of Helicobacter pylori infection: facts or myth? A critical review. J Dig Dis 13: $342-349$.

3. Malfertheiner P, Megraud F, O'Morain CA, Atherton J, Axon AT, et al. (2012) Management of Helicobacter pylori infection--the Maastricht IV/ Florence Consensus Report. Gut 61: 646-664.

4. Fock KM, Katelaris P, Sugano K, Ang TL, Hunt R, et al. (2009) Second Asia-Pacific Consensus Guidelines for Helicobacter pylori infection. J Gastroenterol Hepatol 24: 1587-1600.

5. Rodeghiero F, Stasi R, Gernsheimer T, Michel M, Provan D, et al. (2009) Standardization of terminology, definitions and outcome criteria in immune thrombocytopenic purpura of adults and children: report from an international working group. Blood 113: 2386-2393.

6. Neunert C, Lim W, Crowther M, Cohen A, Solberg L Jr, et al. (2011) The American Society of Hematology 2011 evidence-based practice guideline for immune thrombocytopenia. Blood 117: 4190-4207.

7. Tomiyama Y, Miyakawa Y, Okamoto S, Katsutani S, Kimura A, et al. (2012) A lower starting dose of eltrombopag is efficacious in Japanese patients with previously treated chronic immune thrombocytopenia. J Thromb Haemost 10: 799-806.

8. Kuter DJ, Bussel JB, Lyons RM, Pullarkat V, Gernsheimer TB, et al. (2008) Efficacy of romiplostim in patients with chronic immune thrombocytopenic purpura: a double-blind randomised controlled trial. Lancet 371: 395-403.

9. Stasi R, Pagano A, Stipa E, Amadori S (2001) Rituximab chimeric antiCD20 monoclonal antibody treatment for adults with chronic idiopathic thrombocytopenic purpura. Blood 98: 952-957.

10. Gasbarrini A, Franceschi F, Tartaglione R, Landolfi R, Pola P, et al. (1998) Regression of autoimmune thrombocytopenia after eradication of Helicobacter pylori. Lancet 352: 878.

11. Emilia G, Longo G, Luppi M, Gandini G, Morselli M, et al. (2001) Helicobacter pylori eradication can induce platelet recovery in idiopathic thrombocytopenic purpura. Blood 97: 812-814.

12. Suzuki T, Matsushima M, Masui A, Watanabe K, Takagi A, et al. (2005) Effect of Helicobacter pylori eradication in patients with chronic idiopathic thrombocytopenic purpura-a randomized controlled trial. Am J Gastroenterol 100: 1265-1270. 
13. Asahi A, Kuwana M, Suzuki H, Hibi T, Kawakami Y, et al. (2006) Effect of Helicobacter pylori eradication regimen on anti-platelet autoantibody response in infected and uninfected patients with idiopathic thrombocytopenic purpura. Haematologica 91:1436-1437.

14. Fujimura K, Kuwana M, Kurata Y, Imamura M, Harada H, et al. (2005) Is eradication therapy useful as the first line of treatment in Helicobacter pylori - positive idiopathic thrombocytopenic purpura? Analysis of 207 eradicated chronic ITP cases in Japan. Int J Hematol. 81: 162-168.

15. Takahashi T, Yujiri T, Shinohara K, Inoue Y, Sato Y, et al. (2004) Molecular mimicry by Helicobacter pylori CagA protein may be involed in the pathogenesis of $\mathrm{H}$. pylori-associated chronic idiopathic thrombocytopenic purpura. Br J Haemat 124: 91-96.

16. Hashino S, Mori A, Suzuki S, Izumiyama K, Kahata K, et al. (2003) Platelet recovery in patients with idiopathic thrombocytopenic purpura after eradication of Helicobacter pylori. Int J Hematol 77: 188-191.

17. Kohda K, Kuga T, Kogawa K, Kanisawa Y, Koike K, et al. (2002) Effect of Helicobacter pylori eradication on platelet recovery in Japanese patients with chronic idiopathic thrombocytopenic purpura and secondary autoimmune thrombocytopenic purpura. Br J Haematol 118: 584-588.

18. Jarque L, Andreu R, Llopis L, De la RubiaJ, Gomis F, et al. (2001) Absence of platelet response after eradication of Helicobacter pylori infection in patients with chronic idiopathic thrombocytopenic purpura. Br J Haematol 115: 1002-1003.

19. Michel M, Khellaf M, Desforges L, Lee K, Schaeffer A, et al. (2002) Autoimmune thrombocytopenic Purpura and Helicobacter pylori infection. Arch Intern Med 162: 1033-1036.

20. Campuzano-Maya G (2007) Proof of an association between Helicobacter pylori and idiopathic thrombocytopenic purpura in Latin America. Helicobacter 12: 265-273.

21. Franchini M, Cruciani M, Mengoli C, Pizzolo G, Veneri D (2007) Effect of Helicobacter pylori eradication on platelet count in idiopathic thrombocytopenic purpura: a systematic review and meta-analysis. J Antimicrob Chemother 60: 237-246.

22. Stasi R, Sarpatwari A, Segal JB, Osborn J, Evangelista ML, et al. (2009) Effects of eradication of Helicobacter pylori infection in patients with immune thrombocytopenic purpura: a systematic review. Blood 113: 1231-1240.

23. Emilia G, Luppi M, Zucchini P, Morselli M, Potenza L, et al. (2007) Helicobacter pylori infection and chronic immune thrombocytopenic purpura: long-term results of bacterium eradication and association with bacterium virulence profiles. Blood 110: 3833-3841.

24. Satake M, Nishikawa J, Fukagawa Y, Akashi K, Okamoto T, et al. (2007) The long-term efficacy of Helicobacter pylori eradication therapy in patients with idiopathic thrombocytopenic purpura. J Gastroenterol Hepatol 22: 2233-2237.

25. Kikuchi T, Kobayashi T, Yamashita T, Ohashi K, Sakamaki H, et al. (2011) Eight-year follow-up of patients with immune thrombocytopenic purpura related toH. pyloriinfection. Platelets 22: 61-64.

26. Sato R, Murakami K, Okimoto T, Watanabe K, Kodama M, et al. (2011) Development of corpus atrophic gastritis may be associated with Helicobacter pylori-related idiopathic thrombocytopenic purpura. J Gastroenterol 46: 991-997.

27. Higashi H, Tsutsumi R, Muto S, Sugiyama T, Azuma T, et al. (2002) SHP-2 tyrosine phosphatase as an intracellular target of Helicobacter pylori CagA protein. Science 295: 683-686.

28. Atherton JC, Peek RM Jr, Tham KT, Cover TL, Blaser MJ (1997) Clinical and pathological importance of heterogeneity in vacA, the vacuolating cytotoxin gene of Helicobacter pylori. Gastroenterology 112: 92-99.
29. Yamaoka Y, Kodama T, Gutierrez O, Kim JG, Kashima K, et al. (1999) Relationship between Helicobacter pylori iceA, cagA, and vacA status and clinical outcome: studies in four different countries. J Clin Microbiol 37: 2274-2279.

30. Franceschi F, Christodoulides N, Kroll MH, Genta RM (2004) Helicobacter pylori and idiopathic thrombocytopenic purpura. Ann Intern Med 140: 766-767.

31. Shimizu T, Kusugami K, Ina K, Imada A, Nishio Y, et al. (2000) Helicobacter pylori-associated gastric ulcer exhibits enhanced mucosal chemokine activity at the ulcer site. Digestion 62: 87-94.

32. Veneri D, Gottardi M, Guizzardi E, Zanuso C, Krampera M, et al. (2002) Idiopathic thrombocytopenic purpura, Helicobacter pylori infection, and HLA class II alleles. Blood 100: 1925-1926.

33. Ishiyama M, Teramura M, Iwabe K, Kato T, Motoji T (2006) Clonally expanded T-cells in the peripheral blood of patients with idiopathic Thrombocytopenic purpura and Helicobacter pylori infection. Int J Hematol 83: 147-151.

34. Suzuki T, Matsushima M, Shirakura K, Koike J, Masui A, et al. (2008) Association of inflammatory cytokine gene polymorphisms with platelet recovery in idiopathic thrombocytopenic purpura patients after the eradication of Helicobacter pylori. Digestion 77: 73-78.

35. Asahi A, Nishimoto T, Okazaki Y, Suzuki H, Masaoka T, et al. (2008) Helicobacter pylori eradication shifts monocyte Fcgamma receptor balance toward inhibitory FcgammaRIIB in immune thrombocytopenic purpura patients. J Clin Invest 118: 2939-2949.

36. Yeh JJ, Tsai S, Wu DC, Wu JY, Liu TC, et al. (2010) P-selectin-dependent platelet aggregation and apoptosis may explain the decrease in platelet count during Helicobacter pylori infection. Blood 115: 4247-4253.

37. Suvajdzi Ä N, Stankovï̈ B, Artiko V, Cvejï̈ T, Bulat V, et al. (2006) Helicobacter pylori eradication can induce platelet recovery in chronic idiopathic thrombocytopenic purpura. Platelets 17: 227-230.

38. Sayan O, Akyol Erikci A, Ozturk A (2006) The Efficacy of Helicobacter pylori eradication in the treatment of idiopathic thrombocytopenic purpura--the first study in Turkey. Acta Haematol 116: 146-149.

39. Estrada-Gómez RA, Parra-Ortega I, Martínez-Barreda C, Ruiz-Argüelles GJ (2007) Helicobacter pylori infection and thrombocytopenia: a singleinstitution experience in Mexico. Rev Invest Clin 59: 112-115.

40. Sivapathasingam V, Harvey MP, Wilson RB (2008) Helicobacter pylori eradication: a novel therapeutic option in chronic immune thrombocytopenic purpura. Med J Aust 189: 367-370.

41. Jackson SC, Beck P, Buret AG, O'Connor PM, Meddings J, et al. (2008) Long term platelet responses to Helicobacter pylori eradication in Canadian patients with immune thrombocytopenic purpura. Int J Hematol 88: 212-218.

42. Tag HS, Lee HS, Jung SH, Kim BK, Kim SB, et al. (2010) Effects of Helicobacter pylori eradication in patients with immune thrombocytopenic purpura. Korean J Hematol 45: 127-132.

43. Payandeh M, Sohrabi N, Zare ME, Kansestani AN, Hashemian AH (2012) Platelet Count Response to Helicobacter pylori Eradication in Iranian Patients with Idiopathic Thrombocytopenic Purpura. Mediterr J Hematol Infect Dis 4: e2012056.

44. Gan GG, Norfaizal AL, Bee PC, Chin EF, Habibah AH, et al. (2013) Helicobacter pylori infection in chronic immune thrombocytopenic purpura patients in Malaysia. Med J Malaysia 68: 231-233. 